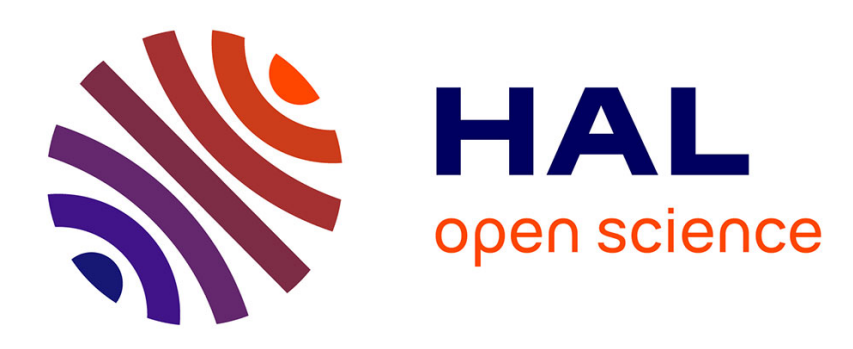

\title{
Does the electric Lehmann effect exist in cholesteric liquid crystals?
}

Alain Dequidt, Patrick Oswald

\section{To cite this version:}

Alain Dequidt, Patrick Oswald. Does the electric Lehmann effect exist in cholesteric liquid crystals?. European Physical Journal E: Soft matter and biological physics, 2007, 24 (2), pp.157-166. 10.1140/epje/i2007-10226-0 . ensl-00202320

\section{HAL Id: ensl-00202320 \\ https://hal-ens-lyon.archives-ouvertes.fr/ensl-00202320}

Submitted on 5 Jan 2008

HAL is a multi-disciplinary open access archive for the deposit and dissemination of scientific research documents, whether they are published or not. The documents may come from teaching and research institutions in France or abroad, or from public or private research centers.
L'archive ouverte pluridisciplinaire $\mathbf{H A L}$, est destinée au dépôt et à la diffusion de documents scientifiques de niveau recherche, publiés ou non, émanant des établissements d'enseignement et de recherche français ou étrangers, des laboratoires publics ou privés. 


\title{
Does the electric Lehmann effect exist in cholesteric liquid crystals?
}

\author{
A. Dequidt ${ }^{\mathrm{a}}$ and P. Oswald \\ Laboratoire de Physique, École Normale Supérieure de Lyon, UMR 5672 of the CNRS, 46 Allée d'Italie, 69364 Lyon, France
}

Received 23 July 2007

Published online: 10 October 2007 - (C) EDP Sciences / Società Italiana di Fisica / Springer-Verlag 2007

\begin{abstract}
Experiments have shown that cholesteric droplets or cholesteric fingers may be put into motion by the action of an electric field. The former rotate whereas the latter drift perpendicularly to their axes. In all cases, the texture moves without visible material transport. The electric Lehmann effect was initially used to interpret these observations but, recently, alternative explanations were found, based on electrohydrodynamics. Another experiment in this area was that of Padmini and Madhusudana (Liq. Cryst. 14, 497 (1993)). Performed in 1993 with a compensated cholesteric liquid crystal under fixed planar boundary conditions, it was also explained in terms of electric Lehmann effect. We conducted the same experiment and extended it to a $\pi$-twisted planar geometry. Although our experimental results agree with those of Padmini and Madhusudana, we demonstrate that they are incompatible with an electric Lehmann effect. By contrast, an explanation based on flexoelectricity allows us to interpret the whole data set obtained in both geometries. The consequence is that there is at the moment no clear experimental evidence of the electric Lehmann effect.
\end{abstract}

PACS. 61.30 .Gd Orientational order of liquid crystals; electric and magnetic field effects on order $-77.65 .-\mathrm{j}$ Piezoelectricity and electromechanical effects - 42.70.Df Liquid crystals

\section{Introduction}

In 1900, Otto Lehmann observed that the texture of particular droplets of a cholesteric liquid crystal spread out between two glass plates could be put into motion when heated from below $[1,2]$. The Lehmann rotation was explained qualitatively 68 years later by Leslie [3], who showed that the chirality allows the existence of an internal torque associated with a heat current. In 1974, de Gennes noticed that an electric current could similarly induce a torque on the director $\mathbf{n}$, of expression [4]

$$
\Gamma_{\text {Lehm }}=-\nu_{E}(\mathbf{n} \times \mathbf{E}) \times \mathbf{n},
$$

thus predicting the existence of an electromechanical coupling with the electric field $\mathbf{E}$ analogous to the thermomechanical Lehmann effect. For this reason, we will speak abusively in the following of "electric Lehmann effect".

In the past, several experiments in cholesteric liquid crystals were initially explained by using this concept, but in most cases another explanation was found.

The first example is that of the "rotating drop" of Madhusudana and Pratibha [5,6]. In their experiment, a flattened cholesteric drop sandwiched between two glass electrodes wetted with a liquid epoxy resin was subjected

\footnotetext{
${ }^{a}$ e-mail: alain.dequidt@ens-lyon.fr
}

to a DC electric field. They observed that the drop texture was spinning clockwise under an upwards-pointing field, and counterclockwise when the field was reversed. They interpreted this continuous rotation in terms of electric Lehmann effect and estimated the corresponding electromechanical coupling coefficient. They found that its sign was positive (respectively negative) for a right- (respectively left-) handed cholesteric. It turns out that another model, only based on classical electrohydrodynamics, was recently proposed by Tarasov and Kramer [7]. According to them, there is no need to introduce a supplementary electromechanical coupling to explain the drop rotation.

The second example deals with cholesteric fingers (CF) (for a review about them, see $[8,9]$ ). The latter form when the liquid crystal is sandwiched between two glass plates treated for strong homeotropic anchoring and when the helical pitch is comparable to the sample thickness. Experiments showed that CFs can form spirals which rotate at constant angular velocity under $\mathrm{AC}$ [10-12] or DC [13] electric field. The spiral formation is mainly due to a drift of the fingers perpendicularly to their axes. A good candidate to explain the drift is the electric Lehmann effect and was indeed proposed by Gil et al. [14,15]. Nevertheless, further experimental investigations showed that the drift was directly related to the ionic force of the sample, 
suggesting an electrohydrodynamic origin [16]. This interpretation was reinforced theoretically in 2003 by Tarasov et al. $[7,17]$ who proposed that electrohydrodynamic effects (induced by Carr-Helfrich charge separation under $\mathrm{AC}$ field and by flexoelectric charge generation under DC field) could describe quantitatively the drift of the fingers. So, it appeared again that there was no need to introduce an electric Lehmann effect to explain the spiral formation.

The aim of this paper is to reanalyze a last experiment of Padmini and Madhusudana [18] designed to prove the existence of the electric Lehmann effect in cholesteric samples with fixed boundary conditions (whereas, in previous experiments of Madhusudana and Pratibha, the director was free to rotate at the boundaries). This experiment was performed with a compensated cholesteric liquid crystal and was conceived to measure the electromechanical Lehmann coefficient $\nu_{E}$ as a function of the spontaneous twist $q_{0}$ of the phase. The main conclusion of their work was that $\nu_{E}$ vanishes and changes sign at the same temperature as $q_{0}$ (the so-called "compensation temperature" $\left.T_{c}\right)$. Although this result was conform to theoretical predictions of Pleiner and Brand [19,20], it seemed to contradict previous experiments of Éber and Jánossy [21], who found that the "classical" thermomechanical Lehmann coefficient was different from 0 at the compensation temperature. Intrigued by these contradictions, we recently redid the experiment of Éber and Jánossy and confirmed their results $[22,23]$. Being thus in disagreement with Pleiner and Brand predictions and with Padmini and Madhusudana findings, we felt it was important to also redo and reanalyze the experiment of Padmini and Madhusudana, while extending it to another geometry. Our results are described below.

The plan of the paper is the following. In Section 2, we recall the principle of the Padmini and Madhusudana experiment and give our experimental results in this case. In Section 3, we reanalyze the data using a theoretical model that takes into account either the Lehmann effect, or the flexoelectricity. In Section 4, we present a new experiment in a $\pi$-twisted planar geometry. It is compared to the models in Section 5. Finally, we draw general conclusions in Section 6.

\section{The Padmini and Madhusudana experiment in parallel planar geometry}

In this experiment, a sample of a compensated cholesteric liquid crystal is prepared between two transparent electrodes treated for parallel planar anchoring. The sample is subjected to an AC electric field and is illuminated with a laser beam at normal incidence. The experiment consists of extracting the first harmonic from the transmitted intensity between crossed polarizers. This harmonic is interesting because it gives information about distortions of the director field which are linear in the applied electric field. Such distortions may be induced by an electric Lehmann torque or by a torque associated with flexoelectricity [24]. In the subsection below we describe our experimental pro- cedure. Useful values of the material constants are given in the following one. Finally, our experimental results are given and compared with those of Padmini and Madhusudana in the last subsection.

\subsection{Experimental procedure}

As in our previous experiment $[22,23]$, we used a mixture of 8OCB (4-n-octyloxy- $4^{\prime}$ cyanobiphenyl from Synthon Chemicals GmbH \& Co.) and of CC (cholesteryl chloride from Aldrich) in proportion 1:1 in weight. This mixture has a compensation temperature $T_{c}$ of $57.6{ }^{\circ} \mathrm{C}$ and a clearing temperature $T_{i}$ of $67^{\circ} \mathrm{C}$. Commercial electrooptic cells from Instec, Inc. were used. They are treated for anti-parallel planar anchoring and have a patterned ITO area, $5 \mathrm{~mm} \times 5 \mathrm{~mm}$ in size. Each cell was filled by capillarity at "high" temperature (typically $50-60{ }^{\circ} \mathrm{C}$ ) to avoid crystallization of the CC and was then placed inside a Mettler oven which was mounted on the stage of a polarizing Leica microscope. A semi-reflecting plate was placed under the condensor of the microscope. It was inclined by $45^{\circ}$ with respect to the microscope optical axis, which allowed us to visualize the sample in normal conditions while illuminating it with a He-Ne laser beam. Thanks to this system, it was possible to displace the sample inside the oven in order that the laser beam did not intersect the spherical balls dispersed between the electrodes to control their gap $d$ within $\pm 0.2 \mu \mathrm{m}$. In addition, the sample was placed between crossed polarizer and analyzer, with the former making an angle of $\pi / 8$ with the direction of the planar anchoring to obtain maximal signal intensity. The laser beam intensity was detected by a photodiode. A Stanford SR850 lock-in amplifier was used to measure the two components $X_{1}$ and $Y_{1}$ of the first harmonic $I_{1}$ of the signal $I(t)$ coming from the photodiode. We recall that $X_{1}=\left|I_{1}\right| \cos \Phi$ and $Y_{1}=\left|I_{1}\right| \sin \Phi$ by denoting by $\Phi$ the phase shift of $I_{1}$ with respect to the sinusoidal applied voltage $V$. As for the the constant component $I_{0}$ of the signal (harmonic 0), it was measured with a Keithley 2000 Multimeter.

\subsection{Useful material constants}

As we are dealing with electric measurements, it was important to characterize the electric properties of our liquid crystal. To this end, we first determined its dielectric constants by measuring the capacitance of planar and homeotropic samples at the compensation temperature. We found $\varepsilon_{\|}=9.4 \pm 0.5$ and $\varepsilon_{\perp}=4.5 \pm 0.5$. From these measurements and others described in refs. [22,23], we calculated the dielectric anisotropy $\varepsilon_{a}=\varepsilon_{\|}-\varepsilon_{\perp}=4.8 \pm 0.3$. We recall that the subscripts $\|$ and $\perp$ refer to the director orientation.

Another quantity of interest is the charge relaxation frequency $f_{c}$ which characterizes the passage from the conducting regime to the dielectric one. Its general expression is $f_{c}=\frac{\sigma}{2 \pi \varepsilon_{0} \varepsilon}$, where $\varepsilon_{0}$ is the vacuum permittivity, $\varepsilon$ the dielectric constant, and $\sigma$ the conductivity. This quantity was obtained by measuring the electrical impedance $Z$ of a 


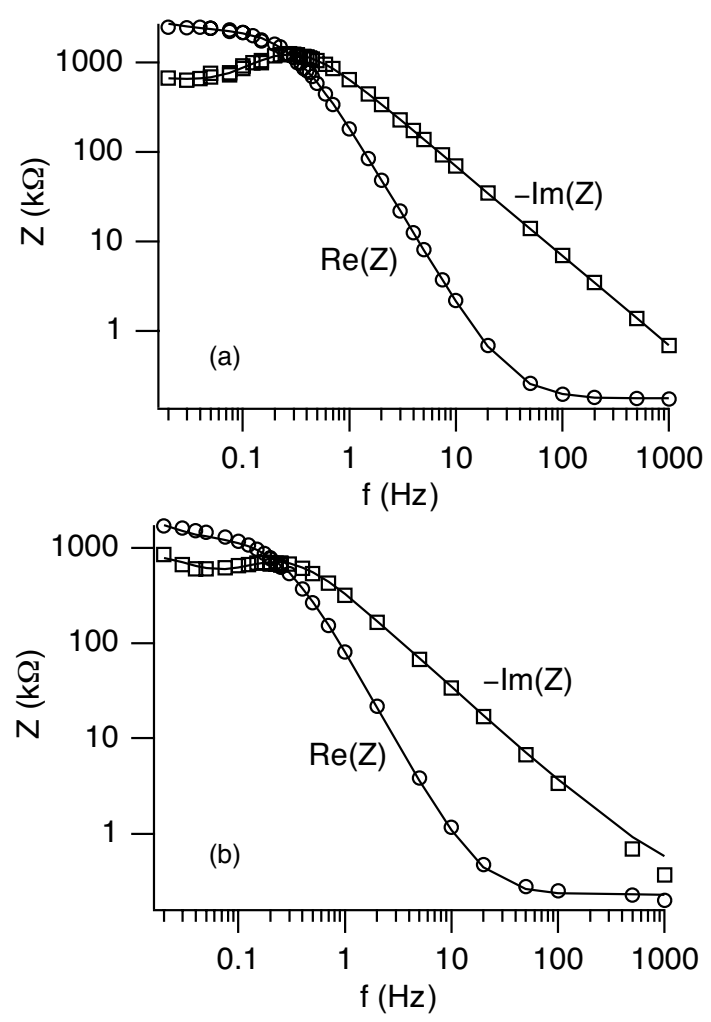

Fig. 1. Real and imaginary parts of the electrical impedance of a $5 \mu \mathrm{m}$ thick planar sample measured at $0.5 \mathrm{~V}$ (a) and at $20 \mathrm{~V}(\mathrm{~b})$.

planar sample at the compensation temperature as a function of the frequency $f=\omega / 2 \pi$. A Hewlett Packard 4284A LCR Meter was used for this measurement. As we can see in Figure 1, this quantity depends on the applied voltage. At low voltage (typically $0.5 \mathrm{~V}$, which is below the onset of the Fréedérickz instability), the sample remains planar so that $f_{c}=f_{c \perp}=\frac{\sigma_{\perp}}{2 \pi \varepsilon_{\perp}}$. On the other hand, at large voltage $(20 \mathrm{~V}$, which is far above the onset of the Fréedérickz instability), the director orients almost homeotropically, so that we now measure $f_{c}=f_{c \|}=\frac{\sigma_{\|}}{2 \pi \varepsilon_{\|}}$. To fit our experimental data, we used the model of Figure 2. In this model, $R_{\text {ITO }}$ is the resistance of the ITO layers, while $R$ and $C$ are, respectively, the resistance and the capacitance of the sample. To account for the Debye boundary layers which form close to the electrodes at very low frequency, a supplementary $\left(R_{D}, C_{D}\right)$ element was introduced [25]. According to this model

$$
\begin{aligned}
Z= & R_{\text {ITO }}+\frac{R}{1+\left(\frac{f}{f_{c}}\right)^{2}}+\frac{R_{D}}{1+\left(\frac{f}{f_{D}}\right)^{2}} \\
& -i\left(\frac{R \frac{f}{f_{c}}}{1+\left(\frac{f}{f_{c}}\right)^{2}}+\frac{R_{D} \frac{f}{f_{D}}}{1+\left(\frac{f}{f_{D}}\right)^{2}}\right),
\end{aligned}
$$

with $f_{c}=\frac{1}{2 \pi R C}$ and $f_{D}=\frac{1}{2 \pi R_{D} C_{D}} \ll f_{c}$. The best fits of the experimental data to this law (solid lines in Fig. 1) gave $f_{c \perp}=291 \mathrm{~Hz}$ and $f_{c \|}=261 \mathrm{~Hz}$, from which we de-

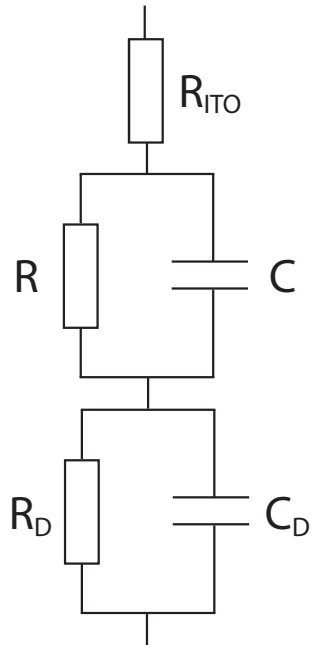

Fig. 2. Electric equivalent model of the sample.

duced

$$
\frac{\sigma_{\|} / \sigma_{\perp}}{\varepsilon_{\|} / \varepsilon_{\perp}}=0.86 \pm 0.03 .
$$

Six other quantities will be necessary in the following: the three Frank elastic constants $K_{i}(i=1,2,3)$, the optical ordinary and extraordinary indices $n_{o}$ and $n_{e}$, and the equilibrium twist $q_{0}$. These quantities were measured in a previous work $[22,23]$. For the first five ones, we found at the compensation temperature:

$$
\begin{aligned}
K_{1} & =(3.4 \pm 0.4) \times 10^{-12} \mathrm{~N}, \\
K_{2} & =(2.8 \pm 0.2) \times 10^{-12} \mathrm{~N}, \\
K_{3} & =(5.9 \pm 0.6) \times 10^{-12} \mathrm{~N} \\
n_{o} & =1.55 \pm 0.01, \quad \text { and } n_{e}=1.64 \pm 0.01,
\end{aligned}
$$

while the equilibrium twist is given as a function of the temperature by the following formula:

$$
q_{o}=a\left(T-T_{c}\right)+b\left(T-T_{c}\right)^{2}
$$

with $a=0.1365 \pm 0.001 \mu \mathrm{m}^{-1} \mathrm{~K}^{-1}$ and $b=0.0028 \pm$ $0.003 \mu \mathrm{m}^{-1} \mathrm{~K}^{-2}$.

Finally, we will need the value of the rotational viscosity $\gamma_{1}$. A classical method to measure it at the compensation temperature (at which the phase has a "nematic" structure) consists first of destabilizing a planar sample and then, of observing the relaxation of the distorted director field after the electric field is switched off. To a first approximation, the response of the director is given by

$$
\theta(z, t)=\theta_{0} e^{-\frac{t}{\tau}} \sin \frac{\pi z}{d}
$$

where $\theta$ is the deviation angle of the director with respect to the plane of the electrodes, $t$ the time, $z$ the coordinate perpendicular to the electrodes, and $\tau$ the relaxation time of expression $\tau=\frac{\gamma_{1} d^{2}}{K_{1} \pi^{2}}$ [8]. Suppose now the sample is placed between crossed polarizers oriented at $45^{\circ}$ relative to the anchoring direction. From formula (3) - which is 


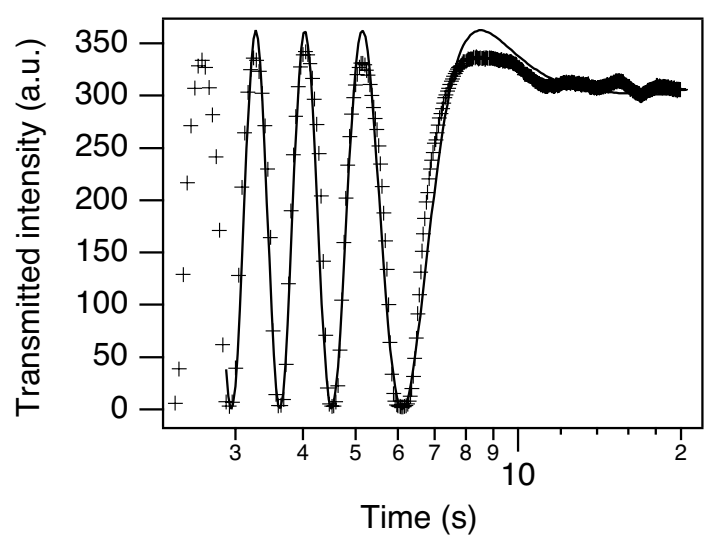

Fig. 3. Fit of the measured transmitted intensity between crossed polarizers (crosses) to the theoretical curve (solid line). The sample was $68 \mu \mathrm{m}$ thick. The fit gave $1 / \tau=0.311 \pm$ $0.01 \mathrm{~s}^{-1}$.

valid when $\theta$ is typically less than $1 \mathrm{rad}$ - we can calculate numerically the intensity of a laser beam crossing the sample at normal incidence. The best fit of the measured intensity to the theoretical one (solid curve in Fig. 3) led to

$$
\gamma_{1}=0.025 \pm 0.003 \mathrm{~Pa} \cdot \mathrm{s} .
$$

\subsection{Experimental results}

All measurements were performed with $5 \mu \mathrm{m}$ thick samples. Harmonics $I_{0}$ and $I_{1}$ (or, equivalently, its components $X_{1}$ and $Y_{1}$ ) were normalized to the value of $I_{0}$ below the threshold of the Fréedérickz instability. We also used the dimensionless voltage $V_{\text {rel }}=\frac{V}{V_{c}}$, where $V_{c}$ is the critical voltage. This quantity characterizes the shift to the threshold of instability. We measured $V_{c}=0.87 \pm 0.01 \mathrm{~V} \mathrm{rms}$. This value is independent of the frequency, except below $40 \mathrm{~Hz}$ where it starts to increase because of the screening of the electric field. In addition, we observed that the samples degraded faster at these frequencies, so we avoided to work below $50 \mathrm{~Hz}$. We first measured harmonics $I_{0}$ and $I_{1}$ as a function of the voltage at a fixed frequency and temperature $\left(f=95 \mathrm{~Hz}\right.$ and $T=55.6{ }^{\circ} \mathrm{C}$, which was about $2{ }^{\circ} \mathrm{C}$ below $\left.T_{c}\right)$. At this temperature, the cholesteric was unwound below the onset of the Fréedérickz transition in spite of the fact that its equilibrium twist $q_{0}$ was different from 0 , close to $-0.27 \mu \mathrm{m}^{-1}$. The frequency $(95 \mathrm{~Hz})$ was chosen in order that the signal be strong enough to be easily measurable and in order that the screening effects associated with the Debye layers be negligible. Figure 4 shows that $I_{0}$ starts to increase above the onset of instability, passes through a maximum when $V_{\text {rel }} \approx 1.4$ and then decreases. The behaviour of $I_{1}$ is more interesting as we show in Figure 5: equal to 0 below the onset of instability, its component $Y_{1}$ strongly increases above the onset of instability with a critical exponent bigger than 1 , while its other component $X_{1}$ remains very close to 0 . This behaviour shows that the first harmonic is in quadrature with the applied voltage $(\Phi \approx \pi / 2)$.

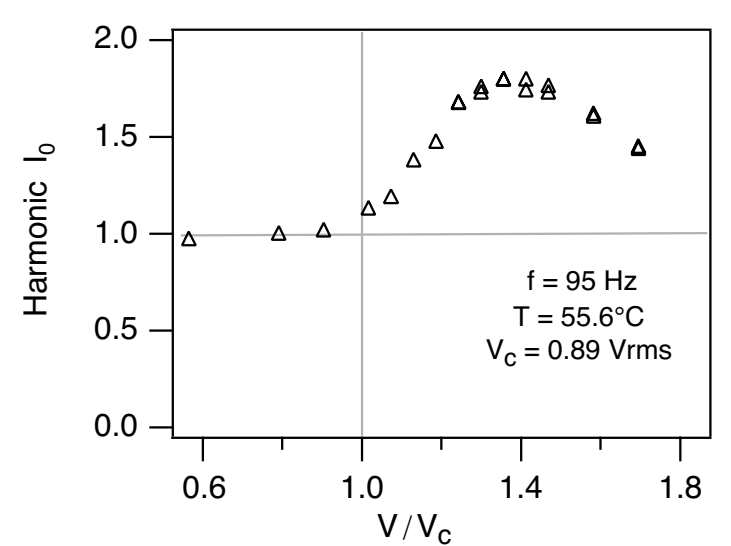

Fig. 4. Normalized harmonic 0 as a function of the relative shift to the critical voltage.

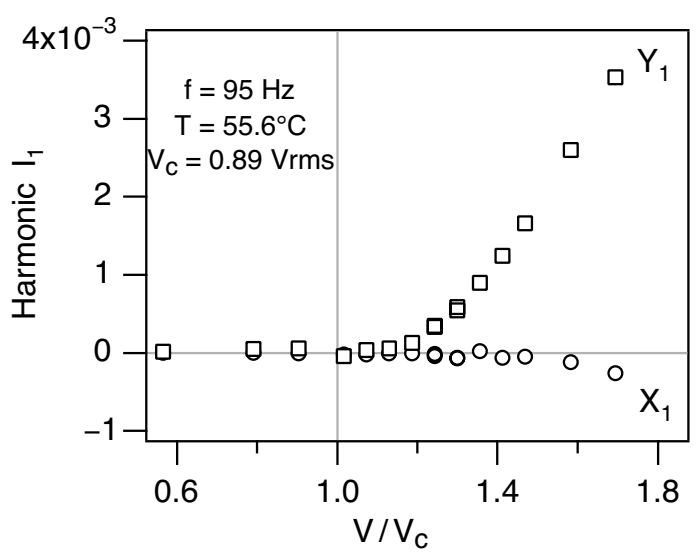

Fig. 5. Normalized harmonic 1 as a function of the relative shift to the critical voltage.

In order to test the influence of the temperature, and thus of the equilibrium twist $q_{o}$, over these results, we measured $X_{1}$ and $Y_{1}$ as a function of the temperature on both sides of the compensation temperature $T_{c}$. The voltage was chosen equal to $1.3 \mathrm{~V} \mathrm{rms,} \mathrm{which} \mathrm{gives} V_{\text {rel }} \approx 1.5$ (independently of the temperature as $V_{c}$ is almost independent of the temperature). In all these experiments, the cholesteric was still completely unwound below the onset of instability. Figure 6 shows that both components $X_{1}$ and $Y_{1}$ vanish and change sign exactly at the compensation temperature (within $\pm 0.1^{\circ} \mathrm{C}$ ). In addition, $X_{1}$ is always very close to 0 , which means that, to a first approximation, the first harmonic is in quadrature with the applied voltage.

Finally, we studied the influence of the frequency $f$. Measurements were again performed at $55.6{ }^{\circ} \mathrm{C}$ and at voltage $1.3 \mathrm{~V} \mathrm{rms}$. Figure 7 shows that the amplitude of the first harmonic $\left|I_{1}\right|$ roughly decreases as $f^{-1}$, while the phase shift remains close to $\pi / 2$.

All these results agree with those of Padmini and Madhusudana. In particular, we confirm that the first harmonic develops only above the onset of the Fréedérickz transition. To a good approximation, this harmonic is in quadrature with the applied voltage and its amplitude 


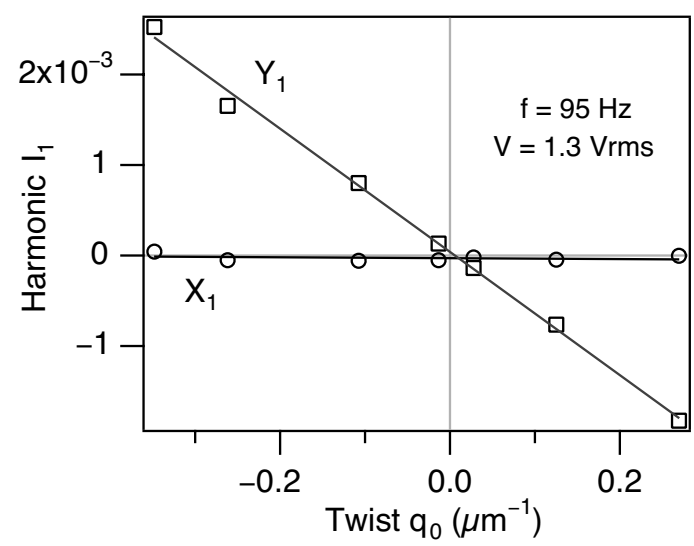

Fig. 6. Normalized harmonic 1 as a function of the spontaneous twist.

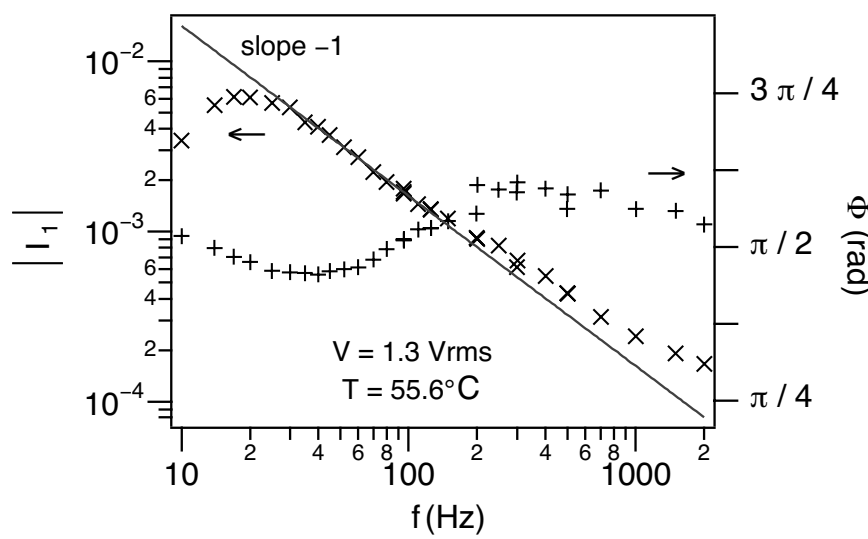

Fig. 7. Normalized harmonic 1 as a function of the frequency.

decreases as $f^{-1}$. And finally, it vanishes and changes sign "exactly" at the compensation temperature (within $\pm 0.1^{\circ} \mathrm{C}$ ), which is a crucial point in this problem.

These results were interpreted by Padmini and Madhusudana as due to an electric Lehmann effect which would disappear at the compensation temperature. Although this interpretation seems reasonable at first sight, we show in the following that it is inconsistent with the experimental data. By contrast, we demonstrate that flexoelectricity is a good candidate to explain the observed phenomena.

\section{Theoretical predictions in unwound geometry}

In this theoretical section, we analyze successively the role of an electric Lehmann torque and of flexoelectricity on the first-harmonic generation. All the calculations were performed using Mathematica. But first let us give the general torque equation we must solve before calculating the transmitted intensity between crossed polarizers.

\subsection{Torque equation}

In this section, we assume that the director field $\mathbf{n}$ is invariant by translation in the horizontal $(x, y)$-plane. The $z$-axis is taken perpendicular to the electrodes. Under these hypotheses (which are verified experimentally in the parallel planar geometry below and above the onset of the Fréedérickz instability), the director has for components:

$\mathbf{n}=(\cos \theta(z, t) \cos \varphi(z, t), \cos \theta(z, t) \sin \varphi(z, t), \sin \theta(z, t))$.

Assuming velocity $\mathbf{v}=0$ (we are always experimentally far below the onset of electrohydrodynamic instabilities and we neglect "backflow" effects), the director obeys the dynamical equation:

$$
\gamma_{1} \mathbf{n} \times \frac{\partial \mathbf{n}}{\partial t}=\mathbf{n} \times \mathbf{h} .
$$

The molecular field $\mathbf{h}$ contains several contributions: an elastic term of expression

$$
\mathbf{h}_{\text {elas }}=\mathbf{h}_{1}+\mathbf{h}_{2}+\mathbf{h}_{3}
$$

with, after setting $q=\mathbf{n} \cdot(\boldsymbol{\nabla} \times \mathbf{n})$ and $\mathbf{R}=\mathbf{n} \times(\boldsymbol{\nabla} \times \mathbf{n})$,

$$
\begin{aligned}
& \mathbf{h}_{1}=K_{1} \boldsymbol{\nabla}(\boldsymbol{\nabla} \cdot \mathbf{n}), \\
& \mathbf{h}_{2}=K_{2}\left[q \nabla \times \mathbf{n}+\boldsymbol{\nabla} \times(q \mathbf{n})-2 q_{0} \boldsymbol{\nabla} \times \mathbf{n}\right], \\
& \mathbf{h}_{3}=K_{3}[\mathbf{R} \times(\boldsymbol{\nabla} \times \mathbf{n})+\boldsymbol{\nabla} \times(\mathbf{n} \times \mathbf{R})]
\end{aligned}
$$

for, respectively, splay-, twist-, and bend-type deformations; follow a dielectric term of expression:

$$
\mathbf{h}_{\text {diel }}=\varepsilon_{0} \varepsilon_{a}(\mathbf{n} \cdot \mathbf{E}) \mathbf{E},
$$

a flexoelectric term given by [26]

$$
\begin{aligned}
\mathbf{h}_{\text {flexo }}= & \left(e_{1}+e_{3}\right)[(\boldsymbol{\nabla} \cdot \mathbf{n}) \mathbf{E}-\boldsymbol{\nabla}(\mathbf{n} \cdot \mathbf{E})] \\
& +e_{3}[2(\mathbf{n} \cdot \boldsymbol{\nabla}) \mathbf{E}-(\boldsymbol{\nabla} \cdot \mathbf{E}) \mathbf{n}],
\end{aligned}
$$

and a Lehmann term of expression

$$
\mathbf{h}_{\mathrm{Lehm}}=\nu_{E} \mathbf{n} \times \mathbf{E} .
$$

For the last term, two hypotheses may be considered: either $\nu_{E}$ is proportional to the equilibrium twist as Pleiner and Brand claim [19], in which case $\nu_{E}=\nu_{0} q_{0}$ (with $\nu_{0}$ a constant), or $\nu_{E}$ is proportional to the local twist $q$ of the director field as proposed by Akopyan and Zel'dovich [27], in which case $\nu_{E}=\nu q$ (with $\nu$ a constant). We emphasize that a constant term $\nu_{C}$ only function of the concentration in chiral molecules could also exist in $\nu_{E}$. On the other hand, we observed that the first harmonic vanishes at $T_{c}$, which implies that this term (if it exists) is not measurable and negligible within our experimental errors [28].

We shall note here that $\mathbf{h}_{\text {diel }}$ is quadratic in the electric field $\mathbf{E}$, contrary to $\mathbf{h}_{\text {flexo }}$ and $\mathbf{h}_{\text {Lehm }}$ which are linear in E. So only the last two terms can lead to first-harmonic generation.

In the following, we will solve the problem in particular cases and make predictions which are compared with the experimental results. 


\subsection{Predictions with a Lehmann coefficient $\nu_{\mathrm{E}}$ proportional to $q_{0}$ (no flexoelectricity)}

Within this hypothesis, the director experiences the Lehmann torque even below the Fréedérickz threshold. It is possible to calculate analytically $\theta$ and $\phi$ by solving equation (5). To a first approximation, we obtain

$$
\begin{aligned}
& \theta(z, t)=A \sin \frac{\pi z}{d}, \\
& \phi(z, t)=\frac{q_{0} d}{4 \pi} A^{2} \sin \frac{2 \pi z}{d}-\nu_{0} q_{0} \frac{E}{\gamma_{1} \omega} \sin \omega t+\phi_{b},
\end{aligned}
$$

where $\phi_{b}$ represents matching terms to boundaries and

$$
A=\frac{2 \sqrt{K_{1}}}{\sqrt{K_{3}-\left(\frac{q_{0} d}{\pi}\right)^{2} K_{2}}}\left\{\begin{array}{cl}
\sqrt{\frac{V-V_{c}}{V_{c}}}, & \text { if } V>V_{c}, \\
0, & \text { if } V<V_{c} .
\end{array}\right.
$$

It is worth noting that when $\theta$ is different from 0 (i.e., above the onset of instability), the director field twists to gain elastic energy on condition that $q_{0} \neq 0$. This explains why the static part of $\phi$ contains a term proportional to $q_{0}$ when $V>V_{c}$. By contrast, the term in $\phi$ due to the Lehmann torque is the same below and above the onset of instability.

The next step was to calculate the transmitted intensity between crossed polarizers. A straightforward calculation using the Jones matrices showed that the amplitude of the first harmonic is maximum when the polarizer makes an angle of $\pi / 8$ with the anchoring direction, in agreement with experiments. Under this hypothesis, we obtained the general formula

$$
\begin{aligned}
\frac{I}{I_{\mathrm{inc}}} & =\frac{1}{2} \sin ^{2} \frac{\psi}{2} \\
- & \sin \frac{\psi}{2} \operatorname{Im}\left(e^{-i \frac{\psi}{2}} \int_{0}^{d} \frac{\mathrm{d} \phi}{\mathrm{d} z} e^{i k \int_{0}^{z}\left(n\left(z^{\prime}\right)-n_{o}\left(z^{\prime}\right)\right) \mathrm{d} z^{\prime}} \mathrm{d} z\right)
\end{aligned}
$$

in which $\psi=k \int_{0}^{d}\left(n(z)-n_{o}(z)\right) \mathrm{d} z$ is the phase shift between the extraordinary and the ordinary rays and $I_{\text {inc }}$ the intensity of the laser.

This formula first allowed us to compute numerically the constant part of the signal $I_{0}$ (harmonic 0 ). This term is independent within an excellent approximation of the Lehmann or flexoelectric torques. It is shown in Figure 8 after normalization to 1 below the threshold of instability. Its behaviour agrees very well with the experimental observations (Fig. 4).

We then calculated the first harmonic $I_{1}$. Its normalized components $X_{1}$ and $Y_{1}$ are shown in Figure 9. To plot this graph, we used the experimental values of the material constants given in Section 2.2. We took additionally $\nu_{0}=-1.4 \times 10^{-14} \mathrm{~kg} \cdot \mathrm{m} \cdot \mathrm{V}^{-1} \cdot \mathrm{s}^{-2}$. This value was chosen in order that the theoretical prediction fits the experimental value of $Y_{1}$ measured $30 \%$ above the threshold of the Fréedérickz instability.

This graph is clearly incompatible with the experimental data. Indeed, it shows that $Y_{1}$ starts to significantly

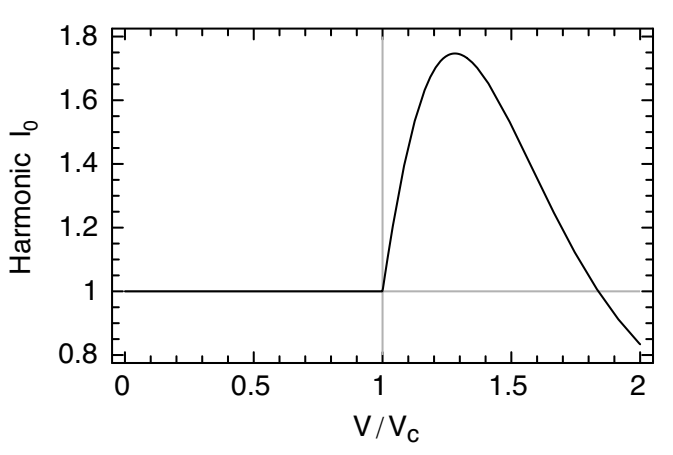

Fig. 8. Calculated harmonic $I_{0}$ as a function of $\frac{V}{V_{c}}$.

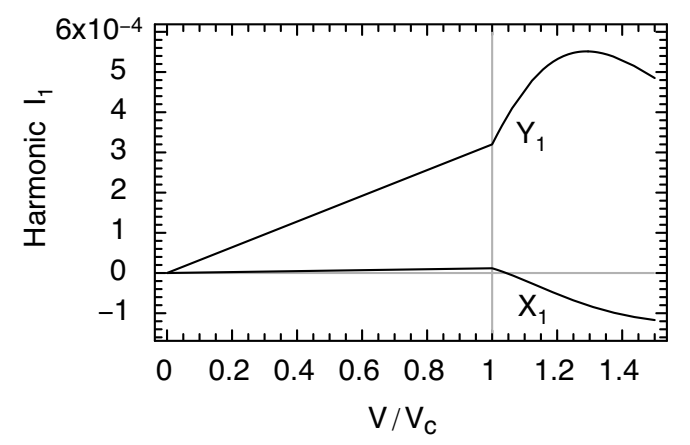

Fig. 9. Calculated harmonic 1 as a function of the voltage assuming that $\nu_{E} \propto q_{0}$.

increase proportionally to the applied electric field from 0 , without important change in order of magnitude at the onset of the Fréedérickz instability.

The immediate implication is that the initial assumption was wrong: the Lehmann coefficient cannot be proportional to $q_{0}[29]$.

This conclusion is in contradiction with the interpretation given by Padmini and Madhusudana [18]. The reason is that these authors assumed without real justification that the plane of polarization of the light was rotating adiabatically below the onset of the Fréedérickz instability, so that the director oscillations were impossible to detect. On the other hand, they interpreted the fact that they could observe the oscillations above $V_{c}$ according to the Mauguin criterion because of the decrease of the birefringence due to the director tilt. Our calculations contradict this interpretation, inasmuch as the change of optical sensitivity is not so important as they expected. As a consequence, we affirm that there is no director oscillations below $V_{c}$.

This conclusion led us to analyze a second hypothesis about the Lehmann coefficient.

\subsection{Predictions with a Lehmann coefficient $\nu_{\mathrm{E}}$ proportional to $q=n \cdot(\nabla \times n)$ (no flexoelectricity)}

Under this new assumption (namely, $\nu_{E} \propto q$, where $q$ is the actual twist $\mathbf{n} \cdot(\boldsymbol{\nabla} \times \mathbf{n})), \theta$ and $\phi$ are exactly null below $V_{c}$ because there is no twist, and thus no Lehmann torque acting on the director. Above $V_{c}$, the static contributions 


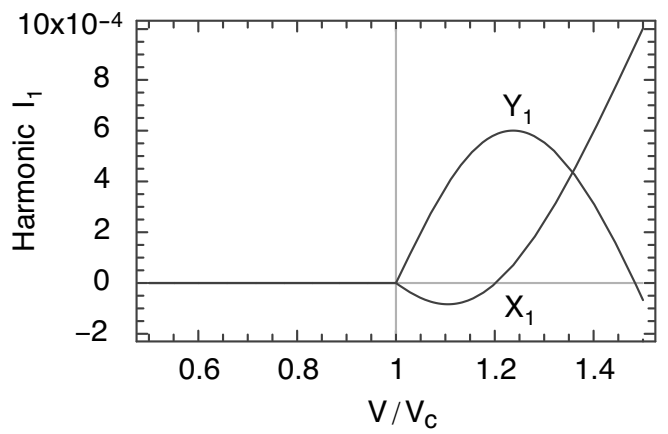

Fig. 10. Calculated harmonic 1 as a function of the voltage assuming that $\nu_{E} \propto q=\mathbf{n} \cdot(\boldsymbol{\nabla} \times \mathbf{n})$.

to $\theta$ and $\phi$ are the same as in the previous calculation. As for the oscillating part of $\phi$, it is due to the Lehmann torque induced by the appearance of a static twist $q$. In complex notation, this part reads

$$
\begin{aligned}
\phi_{1}(z)= & -\frac{i}{2 \gamma_{1} \omega} \nu q_{0} d^{2} E \frac{\kappa^{2} A^{2}}{4 \pi^{2}+\kappa^{2}} \\
& \times\left(\cos \frac{2 \pi z}{d}-\cosh \kappa z+\sinh \kappa z \tanh \frac{\kappa d}{2}\right) e^{i \omega t}
\end{aligned}
$$

with $A$ defined as above and $\kappa=\sqrt{i \frac{\gamma_{1} \omega}{K_{2}}}$.

The corresponding intensity is plotted in Figure 10. Here again, we adjusted the value of $\nu$ in order to match the theoretical and experimental values of $Y_{1}$ found $30 \%$ above the threshold of Fréedérickz instability. This yielded to $\nu=9.3 \times 10^{-13} \mathrm{~kg} \cdot \mathrm{m} \cdot \mathrm{V}^{-1} \cdot \mathrm{s}^{-2}$, which is two orders of magnitude larger than the value of $\nu_{0}$ given previously. This is consistent with the fact that $q \ll q_{0}$ above the onset of instability. As expected, this model is in better agreement with experiments, because it predicts that there is no signal below $V_{c}$. On the other hand, the model does not fit well the experimental data, either for $X_{1}$ or for $Y_{1}$ which are predicted to increase above $V_{c}$ with finite slopes, whereas the slopes are experimentally null (see Fig. 5).

Thus, although this model was better than the previous one, it was not really satisfactory. For this reason, we looked for another explanation based on flexoelectricity. This is what we analyze in the next subsection.

\subsection{Predictions in the presence of flexoelectricity (no Lehmann effect)}

Another linear term comes from the flexoelectric torque. In order that $\mathbf{h}_{\text {flexo }}$ does not reduce to surface terms, we have to take into account gradients of $\mathbf{E}$. They appear naturally above $V_{c}$ when the director field is distorted because of the anisotropy of the electrical conductivities (at $f<f_{c}$ in the conducting regime) or of the dielectric constants (in the dielectric regime at $f>f_{c}$ ). We recall that $f_{c}$ is the charge relaxation frequency defined in Section 2.2. By contrast, $\mathbf{E}$ is constant within the cell gap below $V_{c}$ as the director field is homogeneous. This implies immediately that the first harmonic must be null below $V_{c}$ within this assumption.

Angles $\theta$ and $\phi$ were calculated by solving analytically equation (5), assuming that the electric field was given by the condition $\boldsymbol{\nabla} \cdot \mathbf{j}=0$, where $\mathbf{j}=\tilde{\sigma} \mathbf{E}$ is the current density and $\tilde{\sigma}$ the conductivity tensor. This condition is justified as we are working at $95 \mathrm{~Hz}$ which is smaller than the charge relaxation frequency $f_{c}$ measured in our sample (of the order of $300 \mathrm{~Hz}$, see Sect. 2.2). A straightforward calculation showed that the static components of $\theta$ and $\phi$ have the same expressions as in equations $(10,11)$, excepted that $A$ is slightly modified as follows:

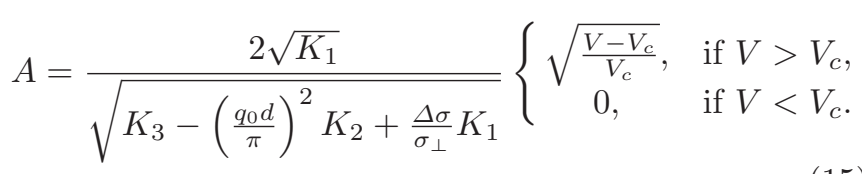

On the other hand, $\theta$ has now an oscillating part at frequency $f$ which reads

$$
\begin{aligned}
\theta_{1}= & \theta_{1}^{*}(t) \sin \frac{2 \pi z}{d} \\
= & \frac{64 A^{3}\left(e_{3}-e_{1}\right) V_{c}}{15 \gamma d^{2}\left(35 \omega_{1}^{2}+4 \omega^{2}\right)} \frac{\Delta \sigma}{\sigma_{\perp}} \\
& \times\left(2 \omega \sin \omega t-7 \omega_{1} \cos \omega t\right) \sin \frac{2 \pi z}{d}
\end{aligned}
$$

with $\omega_{1}=\frac{\pi^{2} K_{1}}{\gamma_{1} d^{2}}$.

As for the oscillating part of $\phi$, it is given by

$$
\phi_{1}=\frac{\omega_{2} A}{\omega_{2}^{2}+\omega^{2}} \frac{q_{0} d}{\pi}\left(\frac{\partial \theta_{1}^{*}}{\partial t}-\omega_{2} \theta_{1}^{*}\right) \sin \frac{\pi z}{d}
$$

with $\omega_{2}=\frac{\pi^{2} K_{2}}{\gamma_{1} d^{2}}$.

We can now discuss the effects of flexoelectricity.

The more immediate one is to induce an oscillation of $\theta$ at frequency $f$ as can be seen from equation (16). At first sight, this should generate a harmonic 1 through the first term of equation (13). In addition, this harmonic should not vanish at $T_{c}$ since it is not proportional to $q_{0}$. Nevertheless, it can be shown that the oscillation of $\theta$ is odd with respect to the middle of the cell (in $\sin (2 \pi z / d)$ ), so that $\psi$ has no harmonic 1 . This is why this term is actually not observed.

However, two indirect effects of the oscillation of $\theta$ arise, which we discuss below.

First, as can be seen in equation (17), the oscillations of $\theta$ are coupled with those of $\phi$, proportionately to $q_{0}$. These oscillations generate a harmonic 1 via the integral in equation (13). Calculations showed that the corresponding signal varies near the onset of instability as $\left(V-V_{c}\right)^{2}$ and decreases with the frequency as $f^{-2}$. This signal is not in quadrature with the voltage.

Second, the oscillations of $\theta$ become detectable also through the integral in equation (13). Indeed, the exponential in factor of $\mathrm{d} \phi / \mathrm{d} z$ contains an oscillating term at frequency $f$ which does not vanish after integration over the thickness. Calculations showed that the corresponding 


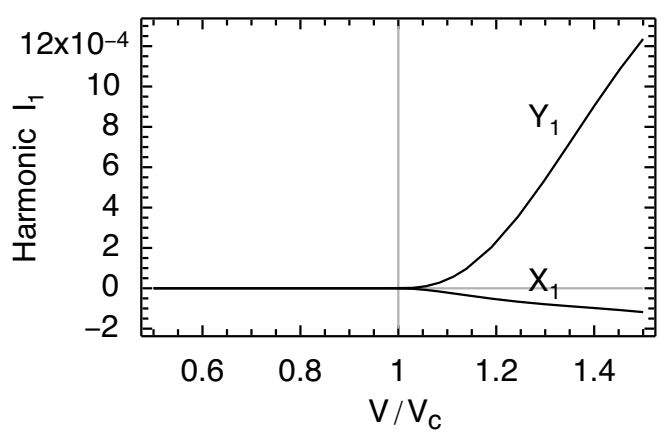

Fig. 11. Calculated harmonic $I_{1}$ as a function of $\frac{V}{V_{c}}$, assuming that the signal is due to a flexoelectric torque.

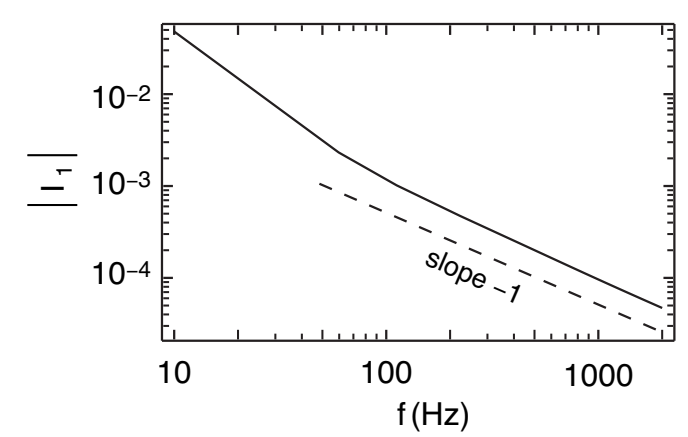

Fig. 12. Harmonic 1 calculated as a function of the frequency at $V=1.5 V_{c}$, assuming that the signal is due to a flexoelectric torque.

harmonic 1 varies in that case as $\left(V-V_{c}\right)^{3}$, is in quadrature with the voltage and decreases as $f^{-1}$. As it is proportional to the static part of $\phi$, it is also proportional to $q_{0}$.

Numerical calculations show that this second contribution to the first harmonic becomes very rapidly much larger than the first one above $V_{c}$. Nevertheless both were kept in the numerics. The calculated intensity is plotted in Figure 11 taking $e_{3}-e_{1}=3 \times 10^{-12} \mathrm{C} \cdot \mathrm{m}^{-1}$. As before, this value was chosen in order that the theoretical and experimental values of $Y_{1}$ coincide at $30 \%$ above the threshold of instability. Note that this order of magnitude for the flexoelectricity anisotropy $e_{3}-e_{1}$ is quite typical for usual liquid crystals [30]. In addition, we calculated the amplitude of the first harmonic as a function of the frequency, assuming that $\boldsymbol{\nabla} \cdot \mathbf{j}=0$ at low frequency $\left(f<f_{c}\right)$ and that $\boldsymbol{\nabla} \cdot \mathbf{D}=0$ (with $\mathbf{D}=\tilde{\varepsilon} \mathbf{E})$ at high frequency $\left(f<f_{c}\right)$. Results are shown in Figure 12. This graph shows that the signal decreases as $f^{-1}$ within a good approximation.

In conclusion, the agreement with the experiment is now much better than in the previous calculation based on the Lehmann effect. Indeed, the calculated component $Y_{1}$ now starts from $V_{c}$ with a horizontal slope and a critical exponent close to 3 . The component $X_{1}$ is negative and small in amplitude with respect to $Y_{1}$ which means that the harmonic 1 is almost in quadrature with the applied voltage. Finally, the amplitude of the harmonic 1 decreases as $f^{-1}$. All these results are in good agreement with experiments.
Nevertheless, we cannot eliminate completely at this level of the discussion the possible existence of a Lehmann effect proportional to the actual twist $q$ of the director field. For this reason, we performed a new experiment in a new geometry susceptible to provide further information about this point. This experiment is described in the following section.

\section{Experiment in twisted geometry}

As we pointed out in the previous section, we cannot completely eliminate the existence of a Lehmann torque proportional to $q$, even if it does not explain the observed phenomena as well as the flexoelectricity does. To know if the Lehmann effect is pertinent in our experiments, we looked for a new geometry allowing us to separate unambiguously the Lehmann torque from the flexoelectric one.

The simplest method was to study the behaviour of the cholesteric twisted by $\pi$ within the cell gap. The same samples as in Section 2 were used for this experiment. In order to achieve the twist condition, it was necessary to change the temperature. We chose to work below $T_{c}$, at $52.5^{\circ} \mathrm{C}$. This temperature was such that the equilibrium pitch was exactly equal to the sample thickness at zero voltage $(d=p / 2)$. Observations and optical intensity measurements were carried out between crossed polarizer and analyzer, with the polarizer at an angle of $\pi / 8$ with respect to the anchoring direction. The frequency of the applied voltage was $95 \mathrm{~Hz}$.

Figure 13 shows the behaviour of the harmonic 0 (normalized to its value below the threshold of instability) as a function of the applied voltage (in V rms). Two curves are visible in this graph. Curve 1 was obtained by increasing the voltage from 0 . Examination of the sample under the microscope showed that a banded texture developed above a critical threshold $V_{c}$ (Fig. 14). This transition was found supercritical as long as the voltage was not increased above a second voltage $V^{+}$. When the voltage was increased above $V^{+}$, the stripes disappeared and the

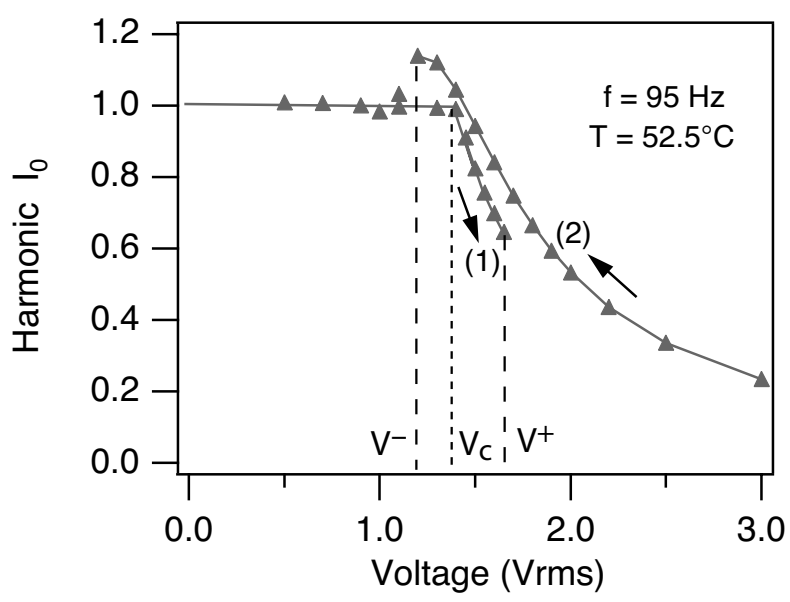

Fig. 13. Normalized harmonic 0 as a function of the applied voltage in twisted geometry. 


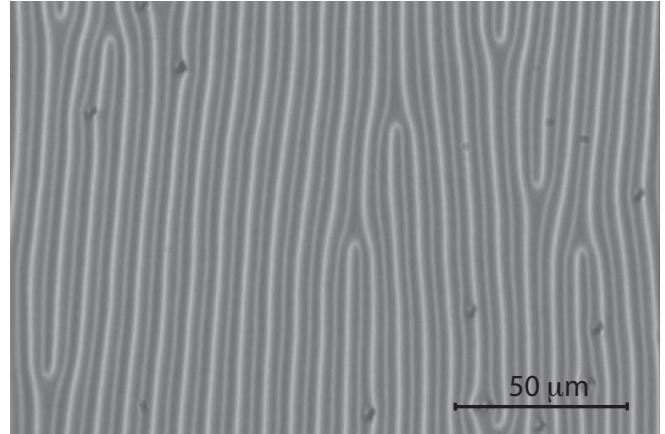

Fig. 14. Banded texture observed above the onset of the Fréedérickz instability in twisted geometry. The bands are parallel to the anchoring direction $\left(d=5 \mu \mathrm{m}, T=52.5^{\circ} \mathrm{C}\right.$, $V=1.6 \mathrm{~V} \mathrm{rms}$ and $f=95 \mathrm{~Hz}$ ).

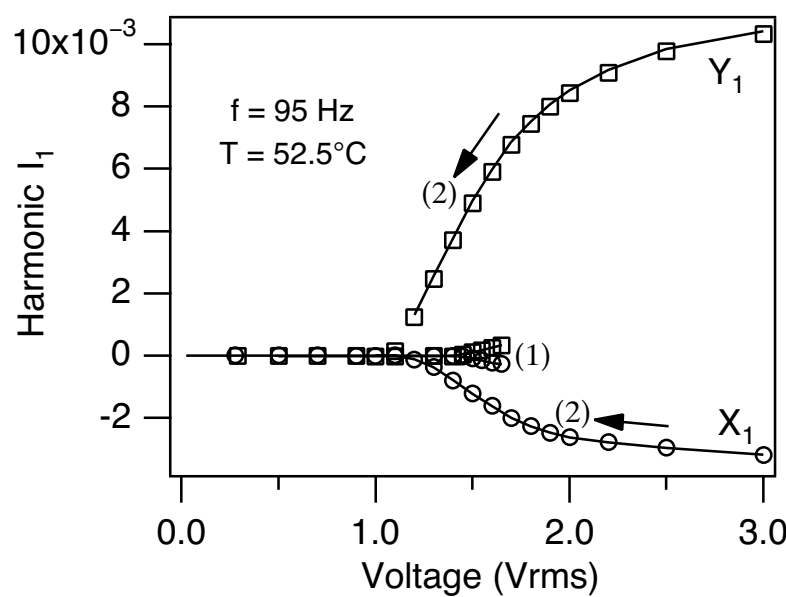

Fig. 15. Normalized harmonic 1 as a function of the voltage.

sample became homogeneous. This abrupt texture change was associated with a jump from curve 1 to curve 2 in Figure 13. Once this new solution was reached, the sample remained homogeneous whatever the applied voltage down to a voltage $V^{-}$at which the intensity jumped again to curve 1. Experimentally, we noticed that $V_{c}$ was very well defined, whereas $V^{+}$and $V^{-}$depended a little on the speed at which the voltage was changed.

These observations showed the existence of two solutions in the twisted geometry: a periodic solution which develops above $V_{c}$ via a supercritical transition and a homogeneous solution which develops at high voltage via a subcritical bifurcation characterized by voltages $V^{+}$ and $V^{-}$.

We also measured the first harmonic of the optical signal associated with the two branches of solution. Data are shown in Figure 15: squares correspond to the component $X_{1}$ and circles to the component $Y_{1}$. As we can see in this graph, the two solutions described above are clearly distinct. But the main point we would like to stress here is that there is no measurable signal below the threshold of instability $V_{c}$ in spite of the fact that the director field is twisted by $\pi$. By contrast, the signal becomes measurable as long as one of the two distorted solutions develops.

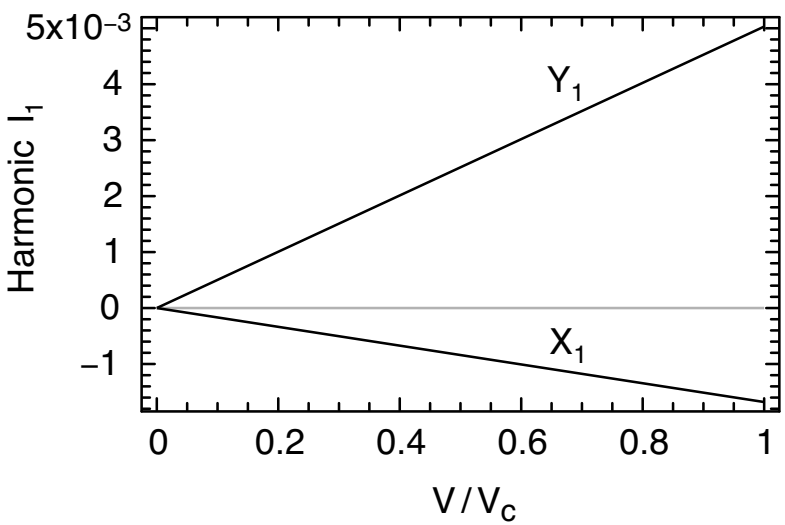

Fig. 16. Predicted harmonic 1 as a function of the voltage below the threshold of the Fréedérickz instability in a $\pi$-twisted cell, in the case $\nu \propto q$.

In the following section, we calculate the two harmonics below the threshold of Fréedérickz instability $V_{c}$.

\section{Theoretical predictions in twisted geometry}

As we pointed out before, there is no flexoelectric effect below the threshold of instability. On the other hand, $q$ is different from 0 whatever the voltage (equal to $\pi / d$ ), so if the Lehmann torque is really measurable, it should also occur below $V_{c}$. Indeed, we calculate that in this case, the angles $\theta$ and $\phi$ are now given by

$$
\begin{aligned}
\theta & =0 \\
\phi & =\frac{\pi z}{d}-\nu \pi \frac{E}{\gamma_{1} \omega d} \sin \omega t+\phi_{b},
\end{aligned}
$$

where $\phi_{b}$ are the matching terms to boundaries. From this solution we calculated the transmitted intensity in the same optical conditions as in the experiment. The calculation was different from that in planar geometry because we could no longer expand $\phi$ in series around 0 . Nevertheless, it remained workable since $\theta$ is equal to zero. Finally, we found for the first harmonic to the first order in $\nu$ and in complex notations

$$
\begin{aligned}
\frac{I_{1}}{I_{\mathrm{inc}}}= & i \pi \nu E \frac{\kappa \psi^{2}}{\gamma_{1} \omega \mu^{2}\left(\kappa^{2} d^{2}+\mu^{2}\right)} \\
& \times\left(\kappa d(\cos \mu-1)+\mu \tanh \frac{\kappa d}{2} \sin \mu\right) e^{i \omega t}
\end{aligned}
$$

with $\mu=\sqrt{4 \pi^{2}+\psi^{2}}$.

To perform the numerics, we used the value of $\nu$ given in Section 3.3. The predicted intensity below the threshold of instability is shown in Figure 16. We notice immediately that the first harmonic should have the same order of magnitude as it had above the threshold in the previous unwound geometry. This prediction is not observed experimentally as we had no measurable signal below $V_{c}$ in twisted geometry. 
We can thus conclude with certainty that the Lehmann torque cannot be responsible for the signal detected above the onset of the Fréedérickz instability in planar geometry (Padmini and Madhusudana experiment) [31].

\section{Conclusion}

We have demonstrated that the absence of a measurable first harmonic below the onset of the Fréedérickz instability in the Padmini and Madhusudana experiment was not due to particular optical conditions (i.e., to an adiabatic rotation of the polarization plane of the light as claimed by these authors) but rather to the fact that there is no measurable Lehmann coefficient proportional to the equilibrium twist $q_{0}$. This result does not confirm the predictions of Pleiner and Brand according to whom the Lehmann coefficient is proportional to $q_{0}$.

Our experiment in twisted geometry allowed us to also exclude a contribution to the Lehmann coefficient proportional to the actual twist of the phase $q$. Such a term was predicted by Akopyan and Zel'dovich, but it is not measurable in the present experiments even if it should exist from our point of view.

Finally, as Padmini and Madhusudana already pointed out, the absence of a measurable first harmonic at the compensation temperature in addition eliminates the constant term in the Lehmann coefficient which should also exist because of the chirality of the molecules (this term should depend on the concentration of chiral molecules). This result is a little surprising because such a term was already observed experimentally by Éber and Jánossy and, very recently, by ourselves, in the thermal case. This comes perhaps from the lack of sensitivity of the technique used in the present work to determine the first-harmonic generation.

To summarize, the experiment of Padmini and Madhusudana and its generalization to the twisted geometry do not allow to detect any electric Lehmann effect.

On the other hand, the first-harmonic generation above the onset of the Fréedérickz instability observed in these experiments can be convincingly explained in terms of flexoelectricity. From our measurements we were also able to determine a value of the flexoelectric anisotropy $e_{3}-e_{1} \approx 3 \times 10^{-12} \mathrm{C} \cdot \mathrm{m}^{-1}$, which is quite compatible with the values given in the literature for other liquid crystals. This point reinforces our interpretation of the observed phenomena.

We thank A. Zywocinsky for the purification of the 8OCB.

\section{References}

1. O. Lehmann, Ann. Phys. (Leipzig) 2, 649 (1900).

2. O. Lehmann, Flüssige Kristalle und ihr Scheinbares Leben (Verlag von Leopold Voss, Leipzig, 1921).

3. F.M. Leslie, Proc. R. Soc. London, Ser. A 307, 359 (1968).
4. P.-G. de Gennes, The Physics of Liquid Crystals (Clarendon Press, Oxford, 1974).

5. N.V. Madhusudana, R. Pratibha, Mol. Cryst. Liq. Cryst. Lett. 5, 43 (1987).

6. N.V. Madhusudana, R. Pratibha, Liq. Cryst. 5, 1827 (1989).

7. O.S. Tarasov, Stuctural Transitions and Dynamics of Liquid Crystals under Flows and Electric Fields, $\mathrm{PhD}$ Thesis, University of Bayreuth (2003).

8. P. Oswald, P. Pieranski, Nematic and Cholesteric Liquid Crystals: Concepts and Physical Properties Illustrated by Experiments (Taylor \& Francis, CRC Press, Boca Raton, 2005).

9. P. Oswald, J. Baudry, S. Pirkl, Phys. Rep. 60, 67 (2000).

10. J.M. Gilli, M. Kamayé, Liq. Cryst. 11, 791 (1992); 12, 545 (1992).

11. M. Mitov, P. Sixou, J. Phys. II 2, 1659 (1992); Mol. Cryst. Liq. Cryst. 231, 11 (1993).

12. P. Ribiere, P. Oswald, S. Pirkl, J. Phys. II 4, 127 (1994).

13. J.M. Gilli, L. Gil, Liq. Cryst. 17, 1 (1994).

14. L. Gil, S. Thiberge, J. Phys. II 7, 1499 (1997).

15. L. Gil, J.M. Gilli, Phys. Rev. Lett. 80, 5742 (1998).

16. J. Baudry, S. Pirkl, P. Oswald, Phys. Rev. E 60, 2990 (1999).

17. O.S. Tarasov, A.P. Krekhov, L. Kramer, Phys. Rev. E 60, 031708 (2003).

18. H.P. Padmini, N.V. Madhusudana, Liq. Cryst. 14, 497 (1993).

19. H. Pleiner, H.R. Brand, Mol. Cryst. Liq. Cryst. Lett. 5, 61 (1987).

20. H. Pleiner, H.R. Brand, Mol. Cryst. Liq. Cryst. Lett. 5, 183 (1988).

21. N. Éber, I. Jánossy, Mol. Cryst. Liq. Cryst. Lett. 72, 233 (1982); 102, 311 (1984); 5, 81 (1988).

22. A. Dequidt, P. Oswald, Europhys. Lett. 80, 26001 (2007).

23. A. Dequidt, A. Zywocinski, P. Oswald, Lehman effect in a compensated cholesteric liquid crystal: experimental evidence with fixed and gliding boundary conditions, submitted to Eur. Phys. J. E.

24. H.R. Brand, H. Pleiner, Mol. Cryst. Liq. Cryst. 292, 141 (1997).

25. L.M. Blinov, Electro-optical and Magneto-optical Properties of Liquid Crystals (John Wiley \& Sons, Chichester, 1983).

26. Note that we have used here the following definition for the flexoelectric polarization: $\mathbf{P}_{\text {flexo }}=e_{1} \mathbf{n} \boldsymbol{\nabla} \cdot \mathbf{n}+e_{3} \mathbf{n} \times(\boldsymbol{\nabla} \times \mathbf{n})$.

27. R.S. Akopyan, B.Ya. Zel'dovich, Sov. Phys. JETP 60, 953 (1984).

28. More precisely, we estimate that $\left|\nu_{C}\right|<8 \times$ $10^{-10} \mathrm{~kg} \cdot \mathrm{V}^{-1} \cdot \mathrm{s}^{-2}$ by taking into account the experimental errors in the measurements of $I_{1}$ as a function of the voltage at $T_{c}$.

29. More rigorously, the proportionality coefficient $\nu_{0}$ must be very small if it exists, less (in absolute value) than $2 \times 10^{-15} \mathrm{~kg} \cdot \mathrm{m} \cdot \mathrm{V}^{-1} \cdot \mathrm{s}^{-2}$ at $55.6^{\circ} \mathrm{C}$ and less than $1 \times$ $10^{-15} \mathrm{~kg} \cdot \mathrm{m} \cdot \mathrm{V}^{-1} \cdot \mathrm{s}^{-2}$ at $59.6^{\circ} \mathrm{C}$. These upper limits were deduced from the analysis of the noise in the measurements of $I_{1}$ below the onset of instability.

30. P.R. Maheswara Murthy, V.A. Raghunathan, N.V. Madhusudana, Liq. Cryst. 14, 483 (1993).

31. More precisely, we can affirm that $|\nu|<6 \times$ $10^{-15} \mathrm{~kg} \cdot \mathrm{m} \cdot \mathrm{V}^{-1} \cdot \mathrm{s}^{-2}$ at $52.6^{\circ} \mathrm{C}$. Again this upper limit was deduced from the analysis of the noise in the measurement of $I_{1}$ below the onset of instability. 